ज્પ FRANÇAISE

$\lessgtr \mathrm{DE}$

딜 PÉDAGOGIE

\section{Revue française de pédagogie}

Recherches en éducation

168 | juillet-septembre 2009

Enseignement et apprentissages, entre psychologie et didactiques

\title{
Entre orthodoxie et pluralisme, les enjeux de l'éducation basée sur la preuve
}

Between orthodoxy and pluralism, the stakes in evidence-based education

Entre ortodoxia y pluralismo, las implicaciones de la educación basada en la prueba

Zwischen Orthodoxie und Pluralismus : die Schwerpunkte einer auf Beweisen

basierenden Erziehung

Frédéric Saussez et Claude Lessard

\section{OpenEdition}

\section{Édition électronique}

URL : http://journals.openedition.org/rfp/1804

DOI : $10.4000 /$ rfp. 1804

ISSN : 2105-2913

\section{Éditeur}

ENS Éditions

\section{Édition imprimée}

Date de publication : 1 juillet 2009

Pagination : 111-136

ISBN : 978-2-7342-1172-3

ISSN : 0556-7807

Référence électronique

Frédéric Saussez et Claude Lessard, «Entre orthodoxie et pluralisme, les enjeux de l'éducation basée sur la preuve », Revue française de pédagogie [En ligne], 168 | juillet-septembre 2009, mis en ligne le 01 juillet 2013, consulté le 19 avril 2019. URL : http://journals.openedition.org/rfp/1804 ; DOI : 10.4000/ rfp. 1804 


\title{
NOTE DE SYNTHÈSE
}

\section{Entre orthodoxie et pluralisme, les enjeux de l'éducation basée sur la preuve}

\author{
Frédéric Saussez et Claude Lessard
}

Cette contribution a pour objet l'approche de l'éducation basée sur des preuves $(E B P)$. Celle-ci se développe autour de la thèse selon laquelle les pratiques et les politiques éducatives devraient être fondées, au nom de l'intérêt public, sur les meilleures preuves scientifiques disponibles. Dans un premier temps, nous caractérisons cette approche et nous dégageons quelques pistes de réflexion visant à rendre intelligible son émergence ; nous identifions également quelquesuns des jalons dans le processus d'institutionnalisation de cette approche en Angleterre et aux États-Unis. En effet, il s'agit d'une approche du changement valorisée, voire même prescrite, par les décideurs politiques des deux côtés de l'Atlantique. Dans un deuxième temps, nous introduisons un schéma d'intelligibilité concernant la dynamique interne du champ de la recherche en éducation reposant sur la tension entre une logique intégratrice et une logique agrégative. Sur cette base, nous essayons de situer l'EBP par rapport à ces logiques, et nous discutons de certains des enjeux liés à l'EBP concernant la recherche, les pratiques professionnelles et la conception et l'implantation des politiques éducatives.

Descripteurs (TESE) : planification de l'éducation, réforme de l'enseignement, recherche en éducation, expert en éducation, politique en matière d'éducation.

\section{L'EBP, UNE APPROCHE DU CHANGEMENT EN ÉDUCATION EN VOIE D'INSTITUTIONNALISATION}

\section{L'EBP, de quoi s'agit-il ?}

L'EBP (ou evidence-based education) est une approche des pratiques et des politiques éducatives qui préconise que celles-ci doivent être fondées sur les données les plus probantes produites par la recherche. Elle vise à réduire l'emprise des modes sur le monde de l'éducation (Slavin, 2008a). En effet, pour les défenseurs de l'EBP, il importe que la chose éducative soit régie par la raison et non 
par la croyance (Davies, 1999) ou l'idéologie (Slavin, 2002, 2008a). Cette approche est présentée comme un système d'aide à la décision rationnelle pour les praticiens et les politiques (Davies, 1999 ; Gough, 2007 ; Oakley, 2004). Nous le montrerons plus loin, I'EBP constitue en fait un vaste projet de transformation en profondeur de la recherche en éducation, de la pratique et des politiques éducatives.

L'approche de l'EBP porte particulièrement sur des questions relatives à l'efficacité d'une intervention ou en d'autres mots par ce qui fonctionne (what works, voir par exemple Gough, 2004). Elle a deux tâches principales : établir la preuve de ce qui fonctionne et rendre cette preuve disponible (Davies, 1999). Pour ce faire, elle s'appuie sur deux dispositifs techniques spécifiques. Le premier consiste en un système de production de la preuve. II s'agit de l'essai contrôlé randomisé (randomized controlled trial). Le second consiste en un système d'analyse, d'évaluation, de synthèse et de diffusion de la preuve : la revue systématique de recherche (systematic review of research). II s'agit en quelque sorte des deux étendards de l'EBP. Nous précisons brièvement ce que recouvrent ces deux dispositifs, sans entrer dans une analyse technique. L'EBP promeut l'usage de l'essai contrôlé randomisé comme mode de production de la preuve. Celui-ci est présenté comme la règle d'or en matière d'évaluation de l'efficacité d'un traitement, d'une méthode ou d'une politique publique. Dans sa plus simple expression, l'essai contrôlé randomisé consiste en un plan expérimental du type :

\section{$\mathrm{R} 01 \mathrm{XO} 2$}

$\mathrm{R} O 1 \mathrm{O} 2$

Ce type de plan expérimental a été popularisé dans le champ de l'éducation par Campbell et Stanley dans un article qui fait toujours autorité en la matière, publié dans le Handbook of research on teaching de 1963 édité par Gage (Fitz-Gibbon, 2003). R signifie que les participants à la recherche ont été versés aléatoirement dans le groupe contrôle et le groupe expérimental. $\mathrm{O} 1$ et $\mathrm{O} 2$ réfèrent aux observations pré-expérimentale et post-expérimentale. Enfin $X$ réfère à une intervention délibérée en vue de produire un effet Y. Campbell et Stanley (1963) ont fait la démonstration que l'essai contrôlé randomisé était un dispositif puissant pour augmenter la validité des résultats obtenus, c'est-à-dire la confiance dans le fait que les différences observées entre les groupes puissent être imputées au traitement expérimental. Oakley (2002) souligne, quant à elle, que la preuve de l'efficacité d'une intervention est établie de manière plus sûre si l'on recourt à une méthodologie expérimentale et idéalement à un essai contrôlé randomisé. II s'agit donc pour les défenseurs de l'EBP du dispositif le plus approprié pour produire des preuves de grande qualité relatives à l'efficacité d'une intervention (Boruch, Moya \& Snyder, 2002).

C'est dans une telle perspective que l'EBP défend l'idée que les politiques éducatives doivent promouvoir des réformes dont l'efficacité a été dûment éprouvée. Ainsi Boruch, Moya et Snyder (2002) plaident pour que chaque nouveau programme ou chaque modification apportée à un programme éducatif soit développé sur une base d'essais cliniques avant d'être adopté à large échelle. Dans un texte qui a contribué à alimenter le débat concernant l'EBP en Angleterre, Hargreaves (1996) a insisté sur le fait que la recherche en éducation devait fournir des preuves concernant l'efficacité d'une intervention éducative en indiquant, par exemple, que si l'enseignant procède de façon X plutôt que X', il y aura une amélioration significative des effets produits. II établissait de la sorte un parallélisme entre le domaine de l'éducation et le domaine médical. Dans cette perspective, il soulignait que les essais contrôlés randomisés constituent, en recherche clinique, le meilleur moyen d'identifier l'impact relatif d'interventions alternatives au regard 
d'une cible prédéterminée. Enfin, pour Slavin (2002, 2008b), cette méthodologie a contribué au développement de la "scientificité " dans la recherche en médecine et dans d'autres domaines tels que l'agriculture, et elle est à la base des progrès fulgurants que ces domaines ont connus dans les dernières décennies.

L'EBP promeut également la revue systématique de recherche (ou RSR) comme mode de gestion des informations évaluatives concernant l'efficacité d'une méthode, d'un programme ou d'une politique. La revue systématique de recherche consiste en un ensemble de procédures formelles pour réunir différents types de preuve, de manière à établir clairement ce que l'on sait d'une méthode et comment elle a été établie (Gough, 2007). Cette démarche est structurée en quatre grandes étapes (Davies, 2004 ; Gough, 2007) :

- formuler une question précise et spécifier les critères de recherche ;

- réaliser une recherche systématique des recherches de première main ;

- évaluer la qualité des preuves établies par la recherche ;

- formuler une réponse claire à la question posée.

La revue systématique de recherche repose sur des règles de procédures pré-spécifiées et des critères transparents pour chacune des décisions prises lors de ces différentes étapes, par exemple les critères d'inclusion et d'exclusion des recherches ou encore les critères d'évaluation de la qualité des recherches sélectionnées (Lessard, 2008). Pour les défenseurs de l'EBP, la transparence des procédures et des critères confère à la revue systématique de recherche toutes les garanties en matière d'objectivité et de neutralité (Gough, 2004). Ainsi la revue systématique de recherche permet d'éviter, grâce à la systématicité et à la transparence de ses procédures, les biais habituels associés aux revues narratives de recherches liés notamment au parti pris du chercheur pour un modèle (Davies, 1999).

La revue systématique de recherche est présentée comme une méthode rigoureuse permettant d'identifier, analyser et évaluer la qualité des résultats produits par la recherche à propos d'une question précise (Davies, 1999 ; Evans \& Benefield, 2001 ; Gough, 2007 ; Oakley, 2002). II s'agit donc d'un dispositif visant à établir la meilleure preuve disponible sur le marché de la recherche à propos d'une question relative à l'efficacité d'une méthode, d'un programme, d'une politique, etc. Elle a pour fonction de fournir l'heure juste aux praticiens, aux politiciens et aux citoyens en mettant à plat les éléments permettant d'apprécier la validité de différentes assertions concernant ceux-ci. Elle a aussi pour fonction d'identifier des zones d'ombre dans la recherche et contribuer de la sorte à ouvrir le terrain à de nouveaux essais contrôlés randomisés. Enfin, les défenseurs de I'EBP présentent la revue systématique de recherche comme un pont entre le monde de la recherche et le monde de la pratique ou de la politique (Hammersley, 2001a), un pont essentiel à l'établissement de bonnes pratiques, c'est-à-dire des pratiques fondées sur la preuve scientifique de leur efficacité (Lessard, 2008). Il apparaît clairement que l'EBP, en promouvant un système spécifique de production et de gestion de la preuve en éducation, met l'accent sur la technique et sur une forme d'objectivité et de neutralité procédurale. L'EBP repose ainsi sur un modèle de rationalité instrumentale au sein duquel la rationalité, la neutralité, la transparence et l'objectivité des décisions sont garanties par un usage systématique de règles de procédure et de méthodes rigoureuses (Biesta, 2007 ; Hammersley, 2001b ; Sanderson, 2003). Nous y reviendrons plus loin.

L'approche de l'EBP ne constitue pas un phénomène isolé. Différents champs de pratiques professionnelles étroitement liées au financement public et au 
développement de politiques publiques ont connu ou connaissent actuellement un tel phénomène, notamment en Angleterre et aux États-Unis : travail social, santé mentale, travail pénitentiaire, soins infirmiers, etc. Différents auteurs (Biesta, 2007 ; Oakley, 2002 ; Reynolds, 2000) mettent en rapport ce mouvement général vers des pratiques fondées sur la preuve avec le succès qu'a connu la médecine basée sur la preuve (MBP) en Angleterre dans les années 1990 et les différentes initiatives privées et publiques qu'elle a inspirées (pour un bref aperçu, voir Reynolds, 2000).

II nous semble pertinent de dégager certaines des caractéristiques de la MBP. En effet, celle-ci sert souvent de modèle de référence aux défenseurs de I'EBP (Hargreaves, 1997 ; Oakley, 2002 ; Slavin, 2002). La MBP a été conçue comme une stratégie pour réduire l'écart entre la recherche et la pratique médicales. En effet, même si la médecine se targue d'être une profession fondée dans une large mesure sur les sciences du vivant, différentes études ont stigmatisé par le passé le faible transfert vers la pratique médicale des résultats de recherche, avec pour conséquence que des pratiques inefficaces, voire dangereuses, étaient encore mises en œuvre et même enseignées, en dépit des avancées de la recherche (Reynolds, 2000). Cochrane constitue la figure de proue de la MBP (Davies, 2004 ; Reynolds, 2000). II a plaidé pour que les ressources allouées aux services de santé soient utilisées pour des traitements ayant démontré leur efficacité. À ce titre, il a été un des principaux promoteurs de l'utilisation des essais cliniques contrôlés randomisés afin d'évaluer l'efficacité d'interventions thérapeutiques. II fut également un des pionniers dans l'utilisation de la revue systématique de recherche afin de rendre disponible pour les praticiens l'information relative aux meilleures preuves disponibles concernant différents traitements. Pour Cochrane (1972, cité par Reynolds, 2000), la preuve produite par la recherche ne se substitue pas à l'expérience du clinicien, mais elle vient alimenter le raisonnement clinique dans lequel ce dernier s'engage pour décider du traitement le plus approprié au patient. Une définition classique de la MBP est d'ailleurs « l'utilisation consciencieuse, explicite et judicieuse des meilleures preuves actuelles pour la prise de décision portant sur le soin des patients individuels, fondée sur des compétences permettant au médecin d'évaluer à la fois l'expérience personnelle et aussi les preuves externes d'une manière systématique et objective » (Sackett et al., 1997 cité par Reynolds, 2000, p. 19).

Dans ce sens, la MBP n'est pas tant une affaire d'évaluation de l'efficacité d'interventions thérapeutiques qu'un modèle normatif de raisonnement clinique. Elle est concernée par la question de savoir comment améliorer l'utilisation de la preuve produite par la recherche, de savoir comment encourager les praticiens à faire un meilleur usage de celle-ci. II s'agit bien de fonder autant que possible les décisions cliniques sur des données probantes issues de la recherche médicale et notamment d'essais contrôlés randomisés ou de revues systématiques de recherche. Ces preuves sont utilisées dans le raisonnement clinique au même titre que l'expérience du praticien. Ainsi la MBP s'appuie, d'une part, sur le développement des méthodes d'analyse de l'épidémiologie en clinique afin de tester l'efficacité d'interventions thérapeutiques (Duc, 2001) et, d'autre part, sur la promotion d'un modèle de raisonnement clinique. La MBP a été popularisée notamment à travers le développement de programmes de formation médicale structurés autour de problèmes à résoudre (problem-based learning). Peile (2000) souligne que l'expression " evidence-based medecine " a été introduite dans le champ médical sous l'effet du développement de l'école de médecine de l'université Mc Master au Canada. Cette expression désignait alors une stratégie d'apprentissage du raisonnement clinique qui a pour point de départ un problème tel qu'il 
est adressé à un médecin par un patient. II s'agit donc d'un modèle d'apprentissage valorisant la recherche, l'évaluation critique et l'incorporation dans la décision clinique des meilleures preuves disponibles concernant le problème et la manière de le résoudre : "Le processus de la MBP est un processus d'apprentissage tout au long de la vie et autodirigé, dans lequel le soin de nos propres patients crée le besoin d'une information clinique importante à propos du diagnostic, du "prognostic", de la thérapie et d'autres enjeux cliniques et de santé, et au cours duquel nous :

1) convertissons ces besoins d'information en questions susceptibles de réponse ;

2) retrouvons, avec une efficience maximale, la meilleure preuve (que celle-ci provienne d'un examen clinique, du laboratoire de diagnostic, de la recherche ou d'autres sources) ;

3) apprécions de façon critique la validité (proximité de la vérité) et l'utilité (applicabilité clinique) de cette preuve ;

4) appliquons les résultats de cette appréciation dans notre pratique clinique ;

5) évaluons notre performance "(Sackett et al., 1997, cité par Lipman, 2000, p. 46).

Pour Trinder (2000), le détour par la MBP a pour intérêt la mise en lumière des différences entre plusieurs versions d'une approche de la pratique basée sur des preuves (ou PBP). Pour cet auteur, plus la référence à une pratique basée sur des preuves s'éloigne du champ de la pratique médicale et d'autres champs proches (médecine vétérinaire, dentisterie, soins infirmiers, etc.), plus elle s'éloigne du modèle promu par la MBP. II y a donc une modification des orientations d'une approche de la pratique professionnelle basée sur des preuves selon les différents champs dans lesquels elle se développe. Dans le même ordre d'idées, Hammersley (2002) indique qu'en médecine, l'accent a été placé principalement sur le transfert des résultats de recherche vers la pratique alors qu'en éducation par contre, l'accent est placé sur l'incapacité de la recherche à produire des preuves satisfaisantes et donc sur la nécessité de transformer la recherche en éducation. On passerait de cette façon d'un projet de transformation de la pratique à un projet de transformation de la recherche.

Dans la même optique, par ce détour, nous souhaitons mettre en lumière en ce qui nous concerne le fait qu'il ne peut y avoir de pratiques basées sur des preuves sans une référence, voire même sans un modèle spécifique de jugement professionnel. Dans le champ médical, le jugement clinique est fortement institutionnalisé et toute tentative de transformation de la pratique médicale doit composer avec cette forme instituée. En d'autres mots, la pratique médicale se caractérise par un noyau technique et une intelligence professionnelle relativement bien définis alors qu'au contraire, en éducation en général et en enseignement en particulier, ceux-ci demeurent relativement flous. Dans la deuxième partie, nous formulerons I'hypothèse d'analyse selon laquelle l'EBP constitue une manière de limiter ce noyau technique à un système de règles visant à réduire et à rendre gérable la complexité au détriment de la prise en compte de ce qui fait l'intelligence professionnelle de l'éducateur, à savoir la gestion de l'incertitude et des dilemmes.

\section{L'EBP, un produit de son temps}

Trinder (2000) souligne, à la suite d'une analyse de l'émergence de l'approche basée sur la preuve dans différents champs de pratiques professionnelles, que cette approche s'est imposée en s'appuyant sur un schème argumentatif 
relativement simple. Celui-ci est fondé sur une thèse difficilement critiquable selon laquelle, pour éviter de porter préjudice au bénéficiaire et de gaspiller les fonds publics, la pratique professionnelle (et les décisions politiques) doit être fondée sur des données probantes, actuelles et vérifiables de l'efficacité des moyens engagés par le professionnel pour résoudre le problème qui lui est soumis. Cette thèse est alors mise en rapport avec différentes idées, qui constituent autant d'arguments, afin de soutenir un processus d'inférence conduisant à l'adoption de la nouvelle thèse selon laquelle l'approche de la pratique basée sur des preuves constitue la solution la plus appropriée pour résoudre les différents problèmes auxquels sont confrontés les praticiens (et les politiciens) et pour être en mesure de fonder leur pratique sur des preuves tangibles.

Le premier argument est l'écart entre la recherche et la pratique, qui est posé comme un fait avéré dont découle la faible, voire même l'absence, d'utilisation des données produites par la recherche par le praticien lorsqu'il effectue un acte professionnel. Ainsi l'activité professionnelle ne repose pas sur un socle rationnel et se fonde alors encore bien souvent sur des convictions, des opinions, des croyances ou d'autres arguments d'autorité. Cet argument est mis en rapport avec l'idée selon laquelle une des stratégies à mettre en œuvre pour dépasser ce problème est de développer des recherches qui sont en prise directe avec les préoccupations des praticiens (et/ou des politiciens) et offrent des réponses précises à des questions concernant ce qui marche. Dans le champ de l'éducation, cette stratégie rhétorique est régulièrement utilisée par les défenseurs de l'EBP (Davies, 1999, 2004 ; Hargreaves, 1997, 1999 ; Slavin, 2002, 2004).

Le deuxième argument est la faible qualité de la recherche et son incapacité à établir la preuve de ce qui marche en raison d'une absence de préoccupation des chercheurs concernant l'utilité pratique de leur recherche (manque de pertinence sociale), leur manque de rigueur et la faiblesse de leurs méthodologies. Cet argument est couplé avec l'idée qu'une stratégie payante pour améliorer la qualité de la recherche dans le champ est d'adopter l'essai clinique contrôlé randomisé présenté comme règle d'or pour établir la preuve de l'efficacité d'une intervention et comme le moteur des progrès fulgurants qu'a connus la médecine (Slavin, 2002). Dans le champ de l'éducation, cette stratégie argumentative a été utilisée par Hargreaves (1997) en Angleterre et Slavin (2002, 2004, 2008b) aux États-Unis. Soulignons qu'en Angleterre des rapports ont été commandés par différentes instances gouvernementales afin de statuer sur la qualité de la recherche en éducation (voir les rapports de Tooley \& Darby, 1998 et Hillage, Pearson, Anderson \& Tamkin, 1998 en Angleterre). Aux États-Unis, le National research council (voir les rapports du NRC, 2002, 2005) a mis sur pied un comité de travail visant à préciser les caractéristiques d'une bonne recherche en éducation à la suite d'un projet de loi avorté visant à définir des critères de qualité pour celle-ci. Le débat fait encore actuellement rage autour de la question de la qualité de la recherche en éducation. Certains évoquent même la fin de la trêve dans la guerre des paradigmes (Lincoln, 2004 ; Bloch, 2004).

Le troisième argument est la quantité croissante d'information à traiter par les praticiens et la difficulté pour ceux-ci de faire le tri dans toute l'information fournie, d'apprécier à leur juste valeur les résultats de recherche et, sur cette base, d'être capable de faire le tri dans la somme considérable des résultats produits par la recherche afin de séparer le bon grain de l'ivraie. Cet argument est couplé avec l'idée qu'il importe par conséquent de développer une méthodologie rigoureuse et objective en mesure de synthétiser de la manière la plus effective possible les différents résultats de recherche concernant les effets d'une intervention afin de les rendre disponibles pour les praticiens. Le mouvement de la pratique 
basée sur des preuves promeut une procédure systématique de revue de la recherche en vue de diffuser la meilleure preuve disponible concernant l'efficacité d'une intervention. Dans le champ de l'éducation, il s'agit d'une des stratégies que les défenseurs de l'EBP valorisent pour transformer les pratiques professionnelles (voir Davies, 1999, 2004 ; Gough, 2004 ; Oakley, 2004). Une des récentes livraisons de la revue Educational researcher est consacrée, sous la direction de Slavin (2008a, 2008b), à une discussion technique concernant les revues systématiques de recherche.

Le quatrième argument est le suivant : des mesures énergiques doivent être prises pour remédier à la situation et favoriser l'adoption de bonnes pratiques par les professionnels. Cet argument est mis en rapport avec l'idée que l'approche de la pratique basée sur des preuves constitue un dispositif méthodologique en mesure de résoudre les différents problèmes discutés précédemment. Si les décideurs souhaitent que la recherche réponde à des préoccupations d'ordre pragmatique en produisant des preuves tangibles relatives à ce qui fonctionne et s'ils souhaitent maximiser les chances que la pratique professionnelle bénéficie réellement des preuves accumulées par la recherche, alors il importe que les décideurs financent massivement le développement d'essais contrôlés randomisés et la confection de synthèses systématiques de recherche. Dans le champ de l'éducation, l'EBP est présentée comme un ensemble de principes et de modes d'opération rationnels pour améliorer la recherche, les pratiques et les politiques éducatives tout en lui assurant une neutralité idéologique (Davies, 1999 ; Slavin, 2002). Bref, au terme d'un tel parcours argumentatif, il est difficile de résister à l'évidence : si l'on souhaite que les pratiques et les politiques éducatives soient effectivement fondées sur la preuve, il est nécessaire alors de financer massivement les différentes stratégies constitutives de l'approche de l'EBP. II importe toutefois de ne pas limiter le contexte d'émergence de l'EBP à une dynamique endogène à différents champs de recherche. Trinder (2000) propose une analyse de différents facteurs sociopolitiques en mesure de rendre compte de l'émergence et du succès de l'approche de la pratique basée sur des preuves.

Bien que l'idée de baser l'éducation ou d'autres formes d'intervention sur des preuves ne soit pas nouvelle (Hammersley, 2001b) et que l'EBP revisite à nouveau frais l'idéal de la modernité visant à ce que les affaires humaines soient gouvernées par la raison et non par la croyance (Davies, 2004), on peut aussi envisager que l'EBP soit bien un produit de son temps (Simons, 2003 ; Trinder, 2000). Pour Trinder (2000), il importe de mettre en rapport l'essor et l'institutionnalisation de l'approche de la pratique basée sur des preuves dans différents champs avec les transformations profondes qui affectent les sociétés modernes occidentales sur les plans politiques, sociaux, économiques, etc. (Beck, 2001 ; Giddens, 1994 ; Jameson, 1991). De manière plus particulière, Trinder (2000) impute le succès de cette approche à sa capacité à endosser et à redéfinir, au sein de son champ, certaines des préoccupations structurant la « société de l'audit » (Power, 1997) et la « société du risque » (Beck, 2001).

II s'agit alors de situer tout d'abord l'approche de l'EBP dans le contexte de différents projets politiques de transformation des services publics engagés aux États-Unis et en Angleterre dans les années 1980 et l'émergence du New public management (NPM) et de l'obligation de résultats (Lessard \& Meirieu, 2004). Sanderson (2003) met d'ailleurs explicitement en rapport l'essor de I'EBP et la modernisation de l'État entreprise par le New Labour en Angleterre. Pour cet auteur, dans un modèle de gestion de l'éducation prônant l'imputabilité, il est essentiel de développer des politiques basées sur des preuves et de mettre en œuvre ce qui effectivement fonctionne. L'émergence du NPM marque la fin de 
l'État providence au profit de l'État gestionnaire. À une logique d'un État interventionniste prenant en charge le développement des secteurs d'intérêt public est substituée une logique minimisant le rôle direct de prestataires de services de l'État dans ces secteurs. On a nommé ce nouvel État de diverses manières : État évaluateur, régulateur, gestionnaire, etc. On aurait tort de penser que l'État disparaît de la scène politique et qu'il laisse la société civile s'autoréguler. II se passe plutôt que l'État se distancie de la livraison ou de la prestation de services qu'il confie au secteur privé ou semi-privé (par exemple, la poste canadienne ou les prisons aux États-Unis) ou assume dorénavant en partenariat avec des entreprises privées des services jusque-là exclusivement publics (par exemple, la gestion des autoroutes). Cela lui permet de réduire la taille de la fonction publique (jugée bureaucratique et inefficace) et de se concentrer sur l'institutionnalisation d'une nouvelle forme de gouvernance, inspirée du NPM, dans un contexte de rationalisation des décisions et de chasse au gaspillage. La conception des buts, du fonctionnement et des modes d'intervention de l'État est profondément remodelée par le NPM (Lessard \& Brassard, 2006). II s'agit notamment pour l'État d'intervenir de manière à soutenir la responsabilisation des individus et des groupes à risque et non plus de pallier sans fin les conséquences de leurs difficultés ou malheurs. II s'agit aussi de rendre les acteurs sociaux responsables de leurs actions. Le NPM introduit l'idée que l'État doit être géré selon les principes de l'entreprise privée. II contribue au développement d'un discours qui met l'accent sur l'efficience, l'efficacité et l'imputabilité. II en découle une réorientation de la demande adressée par l'État au monde de la recherche vers une mesure de l'efficience du fonctionnement de l'État et de l'efficacité de ses interventions. Pour faire bref, l'État doit fonctionner de manière transparente et les contribuables doivent en avoir pour leur argent.

L'EBP partage avec le NPM le mythe de la transparence et la foi en la raison instrumentale. Hammersley (2001b) met en rapport l'insistance sur les caractères explicites, neutres et objectifs des procédures en jeu, par exemple dans les revues systématiques de recherche et l'exigence d'« imputabilité transparente " (ou transparent accountability) valorisée par le NPM. De la même manière que L'EBP se présente comme un système de règles et de procédures rationnelles et neutres permettant d'exclure toute forme de biais et d'assurer l'objectivité des preuves, le NPM se présente comme une stratégie neutre, transparente et surtout rationnelle, permettant de prémunir les décisions politiques de tout risque de conflit d'intérêts ou d'ingérence politique, et de garantir au citoyen qu'il en a bien pour son argent (Trinder, 2000). Dehue (2001) souligne qu'on a assisté à une instrumentalisation de la recherche en sciences sociales par les administrateurs. Celle-ci est en lien avec l'augmentation de la crainte de se voir reprocher des décisions à caractère arbitraire. Cet auteur conçoit d'ailleurs le développement de la pratique basée sur des preuves comme un modèle d'administration. Pour cet auteur, cette pratique ne consiste pas simplement en une question d'application d'une logique et d'une méthode scientifiques, mais en une incarnation des valeurs d'efficacité et d'objectivité centrales au développement du libéralisme au cours du $\mathrm{Xx}^{\mathrm{e}}$ siècle.

Bref, le discours de l'EBP se marie particulièrement bien avec un discours sur l'amélioration de la qualité, l'accroissement de l'efficacité et du niveau de performance ou encore de l'imputabilité. À ce titre, Oancea (2005), à la suite d'une analyse systématique des thèmes, du lexique et des stratégies rhétoriques des trois principaux textes (Hargreaves, 1996 ; Tooley \& Darby, 1998 ; Hillage et al., 1998) qui ont alimenté le débat sur la qualité de la recherche en éducation et participé de cette façon à l'institutionnalisation de l'EBP en Angleterre, a mis en évidence l'usage d'un répertoire caractéristique d'un langage de la gestion et du 
business. Dans le même ordre d'idées, Slavin (2002) exploite habilement le thème de l'imputabilité pour mettre en valeur l'approche de l'EBP : « des politiques éducatives basées sur la preuve seraient importantes en tout temps, mais elles le sont tout spécialement aujourd'hui, étant donné la montée en importance de l'imputabilité » (p. 19).

Au regard du développement de la société de l'audit, l'approche de l'EBP apparaît ainsi comme un produit de son temps. Dans le même ordre d'idées, Power (1997) formule l'hypothèse selon laquelle le développement de la société de l'audit s'inscrit dans un rapport complexe avec le développement de la société du risque (Beck, 2001 ; Giddens, 1994). Cette dernière se caractérise par une anxiété récurrente dans les différents champs de la société. Ce sentiment est lié à un accroissement du sentiment du risque. La société du risque se caractérise également par une diminution de la confiance du grand public dans les experts, ceux-ci étant le plus souvent conçus comme les responsables de l'augmentation des risques et de l'incertitude. L'essor de l'approche de l'EBP est donc à resituer également dans un contexte où il s'agit de concevoir de nouvelles stratégies pour gérer les risques inhérents à une société moderne avancée (Giddens, 1994), caractérisée par la complexité et la nature incertaine et confuse du monde, ainsi que par l'ambivalence du public et des décideurs à l'égard de la science et des experts. En effet, les personnes sont tout à la fois conscientes que la majorité des risques et la plus grande part de l'incertitude qui planent sur la société sont générées par les experts et qu'il est pourtant impossible de se soustraire à une forte dépendance à l'égard de ceux-ci. Pour Giddens (1991, cité par Trinder, 2000), une des stratégies possibles pour gérer cette ambivalence est ce qu'il nomme l'« optimisme raisonnable " (ou sustained optimism). Celle-ci repose sur l'idée que les experts pourront trouver des solutions techniques pour résoudre les problèmes et que la pensée rationnelle offre encore actuellement la meilleure garantie pour assurer une sécurité à long terme. Elle consiste également en quelque sorte à réaffirmer sa foi en la science dans une société du risque. Il s'agit de réinvestir le discours de la modernité en affirmant que le risque, la complexité, l'incertitude peuvent être évalués et contrôlés par les experts ou au moins par les procédures rationnelles mises en place par ceux-ci. Une des stratégies rhétoriques utilisées par les défenseurs de l'EBP est d'ailleurs d'insister sur le fait que cette approche permettra à l'éducation d'accéder enfin à la modernité et de profiter de la sorte de ses bienfaits (Slavin, 2002, 2008a). Sous cet angle, l'approche de l'EBP apparaît comme une stratégie pour rendre gérable la complexité des phénomènes éducatifs ainsi que pour dépasser la méfiance des politiques et du grand public à l'égard de la science, en mettant en avant ses procédures rationnelles, évaluables et transparentes. Celles-ci sont les garantes de la sécurité qu'elle peut apporter aux politiciens, aux praticiens et aux clients en établissant hors de tout doute ce qui marche.

Comme nous l'avons souligné précédemment, l'idée que la pratique professionnelle ou les décisions politiques doivent être basées sur des preuves scientifiques n'est pas nouvelle. Par exemple, Davies (1999) fait référence de manière explicite à Comte et à son projet de science positive. Pour Davies, I'EBP est plutôt un héritage des Lumières et une reprise du projet de chasser la théologie et la métaphysique de la conduite des affaires humaines. Dans le même ordre d'idées, Slavin $(2002,2004,2008 a, 2008 b)$ reprend le discours du combat de la raison avec la foi et métaphoriquement réfère à la nécessité d'éradiquer les différentes idoles (au sens de Bacon) qui minent les potentialités de la raison humaine.

Par ailleurs et toujours en référence à l'histoire des sciences sociales aux États-Unis, nous ne pouvons passer sous silence la référence à Campbell (1969) 
et à son idée de la "société de l'expérimentation » (ou experimenting society). Comme nous l'avons souligné, Campbell a contribué au raffinement des plans expérimentaux en sciences sociales en montrant qu'une répartition aléatoire des sujets dans les groupes expérimentaux et contrôle permettait d'améliorer la validité des résultats (Campbell \& Stanley, 1963). Fitz-Gibbon (2003) rappelle qu'à ce titre, il a joué un rôle considérable dans le développement du champ de l'évaluation. De manière plus particulière, Dehue (2001) souligne que Campbell (1969) était un fervent partisan d'une approche expérimentale des réformes sociales. II défendait l'idée qu'une société intelligente devait traiter ses réformes comme des expérimentations et en contrôler les effets afin de déterminer si elles fonctionnaient ou non et, sur cette base, décider de leur adoption (Dehue, 2001 ; Fitz-Gibbon, 2003). Campbell (1969) concevait le scientifique comme un "serviteur méthodologique de l'experimenting society ", c'est-à-dire une société qui se donnerait les moyens d'utiliser les méthodes des sciences sociales et les procédures d'évaluation afin d'établir rigoureusement les solutions à des problèmes sociaux persistants et d'écarter de cette façon les solutions inefficaces. Davies (2004) montre ainsi la filiation entre les idées de Campbell et les orientations de la Campbell collaboration dont nous parlerons plus loin. Enfin soulignons qu'il reste aussi à analyser l'essor et l'institutionnalisation de I'EBP à la lumière des transformations qui affectent l'activité scientifique et contribuent à l'émergence d'un mode 2 (Gibbons et al., 1994). Ces auteurs ont analysé l'évolution de l'activité scientifique contemporaine en tentant de montrer l'émergence, à côté du modèle scientifique «traditionnel » (le mode 1 de recherche nomothétique, fondamentale, méthodologiquement formalisée, institutionnalisée dans des centres de recherche et des universités, réalisée par des professionnels autonomes et autorégulés), d'un second modèle (le mode 2 de recherche contextualisée, appliquée, axée sur la résolution de problèmes, utilisant des méthodologies variées et adaptées au contexte et aux problèmes étudiés, faite en partenariat avec ses clients ou ses bénéficiaires, ad hoc, en réseau hétéronome et multirégulée). L'EBP constitue-t-elle un prototype du devenir d'une activité scientifique qui combine, dans le champ de l'éducation, des éléments de ces deux types : la rigueur méthodologique du premier et l'hétéronomie du second?

\section{L'EBP, une approche en voie d'institutionnalisation}

Dans cette section, nous allons tenter d'identifier quelques jalons dans le développement et l'institutionnalisation de l'EBP en Angleterre et aux États-Unis. Nous mettons l'accent principalement sur le fait que les orientations de l'EBP et son financement sont intimement liés aux programmes politiques des gouvernements (Atkinson, 2000). Sous cet angle, l'analyse du processus d'institutionnalisation porte son attention plus spécifiquement sur les différentes initiatives gouvernementales, de part et d'autre de l'Atlantique, visant à promouvoir et financer les recherches qui s'inscrivent clairement dans l'approche de I'EBP, soit parce qu'elles produisent des preuves à l'aide d'essais contrôlés randomisés, soit parce qu'elles synthétisent, évaluent et diffusent la meilleure preuve disponible de ce qui marche pour résoudre un problème d'intérêt public précis.

Soulignons d'entrée de jeu que nous formulons à titre d'hypothèse d'analyse qu'un accent différent sur les moyens à mettre en œuvre, ou à tout le moins un poids différent, est placé sur l'un ou l'autre des deux dispositifs techniques, selon que I'on se situe d'un côté ou de l'autre de l'Atlantique. II semble que I'on soit plus proche du modèle de la MBP (Hargreaves, 1997) et de la valorisation de la revue systématique de recherche comme instrument pour développer de meil- 
leures pratiques en Angleterre alors qu'aux États-Unis, I'EBP valorise davantage les essais contrôlés randomisés et s'intéresse peu au jugement professionnel des enseignants. Un peu comme si l'enjeu de l'EBP aux États-Unis se situait au niveau de la conception des politiques éducatives alors qu'en Angleterre, il se situe au niveau de la transformation de la pratique enseignante, pour que celle-ci soit davantage fondée sur la recherche. II s'agit d'une hypothèse à considérer avec prudence au regard notamment de la généalogie distincte de l'EBP aux ÉtatsUnis, où la référence à l'expérimentation sociale et à Campbell sont centrales alors qu'en Angleterre, la référence à la médecine basée sur la preuve et à Cochrane est plus souvent mise en avant.

L'approche de l'EBP a bénéficié d'un contexte particulièrement favorable sur le plan politique pour se développer en Angleterre. En effet, l'evidence-based policy constitue une orientation politique générale du New Labour. Ainsi, l'arrivée au pouvoir de T. Blair s'est traduite par un renouvellement de la foi dans la science et d'une volonté politique d'en faire un usage instrumental (Sanderson, 2003). Gough (2004) mentionne que le livre blanc sur la modernisation du gouvernement défendu par Blair met l'accent sur la nécessité pour la recherche en sciences sociales de produire des preuves concernant l'efficacité des politiques publiques. Comme Blair (1999, cité par Simons, 2003) l'a avancé : “ Ce qui compte, c'est ce qui fonctionne ». Dans le champ de l'éducation, peu avant cela, les rapports commandés par trois agences gouvernementales avaient préparé le terrain en mettant en cause la qualité et la pertinence de la recherche en éducation : le rapport de la Teachers training agency (TTA) et la conférence de Hargreaves (1996), le rapport Tooley (Tooley \& Darby, 1998) réalisé pour l'Office for standards in education (OFSTED) et le rapport Hillage (Hillage et al., 1998) réalisé pour le Department for education and employement (DFEE). Dans ce qui suit, nous analysons ce qui nous apparaît être des jalons importants dans l'institutionnalisation de l'EBP en Angleterre.

Cette dernière s'appuie sur une critique radicale de la qualité et de la pertinence de la recherche en éducation. Pour Hammersley (2002), il importe de ne pas couper cette critique de l'histoire de l'institutionnalisation de la recherche en éducation et de l'universitarisation de la formation des enseignants au $\mathrm{Xx}^{\mathrm{e}}$ siècle et du contre-pied qui s'ensuivit dans les années 1980 et 1990. D'ailleurs, Hammersley (2002) mentionne qu'il y avait déjà eu quelques tentatives de transformer la recherche en éducation au début des années 1990. Rappelons que l'universitarisation de la formation des enseignants avait notamment contribué au développement de la nouvelle sociologie de l'éducation (Trottier, 1987) et que les sociologues britanniques ont, au cours des Trente Glorieuses, développé des approches nettement critiques des politiques éducatives. Cette recherche disciplinaire et critique, ainsi que la formation qu'elle alimenta, furent remises en cause au cours des années 1980 et 1990 par les pouvoirs conservateurs, ceux-ci soutenant qu'elles n'étaient pas pertinentes et ne répondaient pas aux besoins des établissements et des praticiens (Bourdoncle, 1993).

Différents auteurs (Atkinson, 2000 ; Biesta, 2007 ; Hammersley, 2002) qui s'intéressent à l'émergence de l'EBP en Angleterre s'accordent pour faire du rapport de la TTA et de sa présentation par Hargreaves (1996) un moment-clé de celle-ci. Ce dernier se livre à une charge en bonne et due forme contre la qualité de la recherche en éducation et son manque de pertinence. Ainsi il conclut : "On aboutit au final à une recherche éducationnelle franchement médiocre qui ne contribue pas sérieusement à la théorie ou au savoir fondamental ; qui n'est pas (ou peu) pertinente pour la pratique ; qui n'est pas coordonnée avec la recherche antérieure ou postérieure; et qui encombre les revues universitaires que quasiment 
personne ne lit " (Hargreaves, 1996, p. 7). Pour cet auteur, si les enseignants ne font pas usage de la recherche, ce ne sont pas eux qu'il faut blâmer, mais plutôt les chercheurs. Ceux-ci sont incapables de produire des résultats fiables utilisables par les enseignants. II établit un parallèle entre le travail du médecin et celui de l'enseignant. II introduit alors l'idée que la recherche doit produire des preuves concluantes pour que, si un enseignant opte pour X plutôt que X' dans sa pratique professionnelle, alors il y aura une amélioration significative et récurrente du résultat. La règle d'or pour l'établissement de liens de cause à effet consiste, comme nous l'avons mentionné précédemment, à développer des essais contrôlés randomisés. Hargreaves (1996) propose enfin de repenser le financement de la recherche en le soumettant à un plus grand contrôle de la part des praticiens et des politiques, ainsi que de financer des recherches conduites par les enseignants afin de développer la profession en une profession fondée sur la recherche. En fait, Hargreaves a préparé le terrain pour les enquêtes concernant la qualité de la recherche, entreprises sous l'égide de I'OFSTED et du DFEE.

Le rapport Tooley (Tooley \& Darby, 1998) repose sur une analyse critique de 41 articles extraits de 4 revues scientifiques. II conclut que plus de la moitié des articles analysés souffrent de nombreuses carences au niveau méthodologique et qu'ils témoignent de prises de position politique ou idéologique. Ce rapport est présenté par Woodhead (le directeur de l'OFSTED) comme faisant état de la nonpertinence de la recherche en éducation et du gaspillage de fonds publics qu'elle représente. II est pertinent de souligner que Tooley est chercheur à l'université de Manchester mais aussi directeur d'éducation dans un think tank de droite : l'Institute of economic affairs.

Le rapport Hillage (Hillage et al., 1998) porte plus particulièrement sur l'utilité sociale de la recherche en éducation et notamment sa pertinence au regard de l'école (Atkinson, 2000). La recherche en éducation y est présentée comme étant non pertinente et de faible qualité. De plus, elle est difficilement accessible pour les praticiens ou les politiciens. Ce rapport plaide pour la constitution d'un forum indépendant avec des représentants du secteur de la recherche en éducation, des enseignants, des représentants des différentes agences gouvernementales et des membres des différents fonds de recherche. Son mandat serait de définir une stratégie au niveau national et de fournir des orientations pour le développement de la recherche. Ici encore, la question du gaspillage des fonds publics dans une recherche de faible qualité accompagne la diffusion du rapport (Hammersley, 2002).

En outre, l'EBP a constitué en Angleterre une orientation politique générale et différentes initiatives gouvernementales ont été favorables à son institutionnalisation. Ces différentes critiques ont été reprises au niveau politique où elles ont donné lieu à différentes initiatives visant à corriger la situation (Atkinson, 2000 ; Hammersley, 2002 ; Normand, 2006). Il s'agit pour Clark (1998, cité par Hammersley, 2002), alors sous-secrétaire d'état au DFEE, de revitaliser la recherche en éducation à travers le financement de centres d'excellence afin d'encourager des revues critiques de la littérature, des réplications de résultats de recherche et, lorsque cela est approprié, de procéder à des essais contrôlés randomisés. II s'agit, en conformité avec l'orientation générale des politiques publiques du New Labour, d'encourager la recherche en mesure d'identifier les approches éducatives les plus efficaces permettant d'accroître les standards aux différents paliers du système d'éducation. Hammersley (2002) et Normand (2006) mettent en exergue trois initiatives:

- la mise en place en 2000 et le financement de l'Evidence for policy and practice information and coordinating center (ou EPPI center) ; ce centre est 
établi à l'Institute of education de l'université de Londres. II a pour but d'établir et de coordonner des groupes de chercheurs qui souhaitent développer des revues systématiques de recherche concernant des questions d'intérêt public, de fournir une infrastructure afin d'assurer la formation et le développement du savoir-faire, de développer un système standardisé pour collecter et classifier les références bibliographiques et pour initier, et maintenir à jour, les revues, ainsi que de développer des modes de collaboration avec les différents groupes d'utilisateurs (Oakley, 2002) ;

- la mise en place du National educational research forum (NERF) qui a pour mission d'établir des priorités en matière de financement de la recherche en éducation de manière à ce qu'elle soit plus en phase avec les besoins de ses utilisateurs. Le NERF a produit un rapport en 2001 qui trace des orientations pour la recherche afin qu'elle participe au développement de l'EBP. II insiste notamment sur le développement d'un système visant à synthétiser et diffuser la recherche ;

- l'établissement du programme «Teaching and learning » au sein de l'Educational science research council. Ce programme est financé à l'aide de ressources initialement destinées aux départements d'éducation (Hammersley, 2002) ; il vise à développer des recherches ayant un intérêt pratique immédiat permettant de contribuer à une amélioration des résultats scolaires des élèves.

Ces différentes initiatives s'inscrivent dans une visée de développement d'une EBP. David Blunkett (2000, cité par Evans \& Benefield, 2001, p. 527) alors secrétaire d'état au DFEE affirmait que la recherche en sciences sociales devait s'intéresser à des questions directement reliées à des débats politiques ou à des questions de mises en œuvre de politiques sociales : "la science sociale doit être au cœur de l'élaboration des politiques [...] Nous avons besoin des sciences sociales pour aider à déterminer ce qui fonctionne et pourquoi, et quels types d'initiatives politiques ont les meilleures chances d'être efficaces ". II va sans dire que si l'EBP sonne comme de la musique aux oreilles des politiques intéressés par ce qui fonctionne, de telles déclarations politiques sont habilement récupérées par les défenseurs de I'EBP. En effet, la meilleure façon d'établir la preuve de l'efficacité d'un traitement, c'est l'essai contrôlé randomisé. Le débat autour de l'EBP fait encore actuellement rage en Angleterre (voir la presidential adress de Whitty (2006) lors du congrès de la British educational research association). II y a d'autres signes de I'institutionnalisation de cette approche. Ainsi la création par l'EPPI center d'une nouvelle revue en éducation : Evidence \& policy. Soulignons aussi le développement de la Campbell collaboration et son partenariat avec la Cochrane collaboration. Enfin, par le biais du Curriculum, evaluation and management center de l'université de Durham, il se développe l'Evidence-based education network (1). II s'agit d'une initiative qui vise les enseignants. Elle promeut ce qui marche en éducation et elle regroupe les personnes qui partagent les orientations défendues dans un manifeste pour l'EBP (2) : par exemple, l'idée que l'éducation « est trop importante pour que l'on autorise qu'elle soit déterminée par l'opinion non fondée, que celle-ci soit de politiciens, d'enseignants, de chercheurs ou de quiconque " ou encore l'idée qu'il importe de développer une culture professionnelle chez les enseignants au sein de laquelle «la preuve est valorisée plus que l'opinion ", reléguant du même coup au niveau préscientifique tout ce qui n'est pas basé sur la preuve.

Normand (2006) inscrit le développement de l'approche de l'EBP aux ÉtatsUnis dans la perspective des changements introduits dans les politiques évaluatives au niveau fédéral à la fin des années 1970. II se manifeste alors un intérêt pour les essais contrôlés randomisés. Tröhler (2006) montre pour sa part qu'un 
nouveau discours éducatif se met en place à la fin des années 1970. Celui-ci est empreint de vocabulaire économique. II se concrétise notamment par la publication du rapport $A$ nation at risk (États-Unis, 1983). L'idée que la recherche en éducation devrait être en mesure d'indiquer aux praticiens et aux politiques ce qui fonctionne commence à circuler (voir par exemple Bennett, 1986). À partir des années 1990, on observe une augmentation du financement octroyé par le département fédéral d'éducation destiné à des recherches conduites sous la forme d'essais contrôlés randomisés (Slavin, 2002, 2008a) dont le Success for all. Slavin (2002) voit des signes encourageants pour le développement de l'approche de l'EBP dans le financement à concurrence de 150 millions de dollars attribué par le Congrès afin de doter les écoles des fonds nécessaires pour adopter des "modèles éprouvés de réforme ". Slavin (2002) se réjouit de voir la preuve définie en référence à des programmes expérimentaux basés sur la comparaison entre un groupe expérimental et un groupe contrôle. C'est toutefois après 2000 que l'approche de l'EBP monte en puissance aux États-Unis. Elle bénéficie notamment de l'attitude critique des politiques à l'égard de la qualité de la recherche en éducation, qui se matérialise par le dépôt d'un projet de loi au Congrès visant à définir les critères de qualité d'une recherche scientifique en éducation et à contribuer de cette façon à améliorer la qualité de la recherche (NRC, 2002 ; Normand, 2006). Ce texte plaide pour un accroissement de la rigueur de la recherche en éducation. II introduit notamment l'idée que les plans expérimentaux et les essais contrôlés randomisés constituent des stratégies pour atteindre cet objectif (Boruch \& Mosteller, 2002). Ce projet de loi n'a pas abouti. II a toutefois donné lieu à différentes initiatives. Ainsi le NRC a mis sur place un comité ayant pour mandat de clarifier la nature de l'investigation scientifique en éducation et d'identifier des stratégies susceptibles de permettre aux agences fédérales de soutenir la recherche de haute qualité en éducation (NRC, 2002). Par ailleurs, ce projet a contribué également à la mise en place d'un groupe de travail financé par le Council for excellence in government qui est un organisme à but non lucratif dédié à l'amélioration de la performance des gouvernements. Ce groupe est à la base de la création de la coalition pour des politiques éducatives basées sur la preuve en 2001.

Enfin l'adoption de la loi No child left behind (ou NCLB, voir États-Unis, 2001) constitue sans doute un facteur décisif dans l'institutionnalisation de l'EBP aux États-Unis. En effet cette loi affirme la volonté du Congrès de lier le financement de la recherche en éducation au fait qu'elle corresponde au canon de la recherche scientifique. Dans ce texte de loi, la recherche scientifique en éducation est définie en référence à la mise à l'épreuve d'hypothèses et à l'utilisation de plans expérimentaux ou quasi expérimentaux et, lorsque cela est possible, à des essais contrôlés randomisés (Eisenhart \& Towne, 2003 ; Lather, 2004). À ce titre, soulignons que le plan stratégique 2002-2007 du département d'éducation américain énonçait l'objectif de transformer le champ de l'éducation en un champ basé sur la preuve : "À la différence de la médecine, de l'agriculture et de la production industrielle, le champ de l'éducation opère largement sur la base de l'idéologie et du consensus professionnel. En tant que tel, il est assujetti, soumis à des modes et est incapable du progrès cumulatif qui découle de l'application de la méthode scientifique et de la collecte systématique et de l'utilisation d'une information objective dans l'élaboration des politiques. Nous allons changer l'éducation pour en faire un champ fondé sur la preuve " (États-Unis, 2002b, p. 50). Dans le même document, l'accent est placé sur les essais contrôlés randomisés en mesure de fonder des relations causales et d'établir les interventions qui fonctionnent le mieux. Par la suite, comme le mentionnent Eisenhart et Towne (2003), le département d'éducation via l'OERI (Office of educational research and improvement) a 
décidé d'attribuer 18,5 millions de dollars au développement du WWC (What works clearing house) afin d'évaluer et de présenter la force et la nature de la preuve scientifique concernant l'efficacité de différents programmes en éducation, produits et pratiques prétendant améliorer la performance des élèves. Le WWC a notamment pour mission de fournir aux décideurs politiques l'information dont ils ont besoin pour opérer des choix guidés par la meilleure recherche scientifique disponible (États-Unis, 2002a, cité par Davies, 2004, p. 29). En fait, ce financement a été attribué à la Campbell collaboration pour créer un partenariat avec l'American institute for research qui est chargé de l'animation du site du WWC.

La Campbell collaboration est un réseau international de chercheurs en sciences sociales et en analyses des politiques. Le réseau a pour mission d'aider les décideurs politiques, les professionnels et le grand public à prendre des décisions éclairées concernant des interventions efficaces (Davies, 2004). Pour ce faire, ce réseau a pour fonction de préparer, maintenir et disséminer les résultats de revues systématiques de recherche concernant l'efficacité d'interventions dans les champs de l'éducation, de la criminalité, de la justice et du social welfare. Ce réseau existe depuis 1999 (Davies, 2004) et fonctionne selon le modèle de la Cochrane collaboration qui s'est développée dans le champ de la médecine et qui contribue au développement de la médecine basée sur la preuve. Boruch sert de lien entre ces différentes organisations. En effet, en plus d'être un des fondateurs de la coalition pour l'EBP, il est membre de l'American institute for research et de la Campbell collaboration. Notre analyse de l'institutionnalisation de I'EBP en est à ses débuts. II reste de nombreux points à approfondir et notamment le rôle des fondations dans l'essor de cette approche, leurs ramifications ou interactions avec les champs politiques, universitaires et les organisations à but non lucratif. Ainsi par exemple, Diane Ravitch, historienne de l'éducation de l'université de Columbia de New York, est rattachée à la Fordham foundation ; elle est une proche de Chester Finn et, comme lui, elle a été anciennement haut fonctionnaire au département d'éducation américain. Elle est une des fondatrices de la coalition pour l'EBP. Aussi la Brookings foundation a soutenu financièrement une rencontre internationale entre chercheurs spécialisés dans les essais contrôlés randomisés qui a donné lieu à la publication de l'ouvrage de Boruch et Mosteller (2002).

Lather (2004) et Hursch (2007) soulignent que ces fondations ont joué un rôle important dans le consensus politique autour du NCLB. Lather (2004) mentionne en outre que les think tanks conservateurs ont redéployé une partie de leur intérêt et de leur financement vers des questions d'éducation depuis la moitié des années 1980. À ce titre, Davies, Nutley et Smith (2000) indiquent que le développement de I'EBP en Angleterre est à mettre en rapport avec l'accroissement du nombre d'organisations qui cherchent explicitement à conseiller ou à influencer le gouvernement dans son action. Sous cet angle, il reste donc encore du travail à accomplir. Lessard (2006) a déjà tenté de déblayer ce terrain eu égard au rôle des think tanks dans le débat américain sur la formation universitaire des enseignants. II y a aussi des recouvrements possibles entre l'EBP et le school effectiveness (Trinder, 2000). Aux États-Unis, Slavin et Mosteller sont proches de ce mouvement, en Angleterre, Hargreaves est connu pour son engagement envers cette approche. Enfin Normand (2006) souligne que l'EBP est déjà solidement établie au sein de I'OCDE. Le CERI a d'ailleurs organisé une série de conférences visant à promouvoir cette approche, dont la première s'est déroulée à Washington en 2004, sous la houlette du département d'éducation américain.

En résumé, la recherche en éducation a été la cible de virulentes critiques de part et d'autre de l'Atlantique. Celles-ci ont eu diverses répercussions sur la scène politique et notamment la valorisation d'une forme de recherche centrée sur 
l'identification et l'appréciation de ce qui marche. On observe que, depuis la fin des années 1990, d'importants fonds publics sont investis, autant en Angleterre qu'aux États-Unis, dans des programmes de recherche visant à établir l'efficacité d'une méthode, d'un programme ou d'une intervention éducative ainsi que dans la production de revues systématiques de recherche. En outre, des fonds publics sont attribués à des organismes à but non lucratif afin qu'ils jouent le rôle d'interface entre la recherche et diverses catégories d'utilisateurs de la preuve et qu'ils répondent clairement à la question désormais au sommet de l'agenda politique en éducation : quelle est la meilleure preuve disponible sur le marché de la recherche à propos d'une méthode, d'un programme ou d'une intervention éducative ?

\section{TROIS ENJEUX DANS L'INSTITUTIONNALISATION DE L'EPB}

Dans cette partie, nous adoptons une posture d'ami critique de l'EBP. En effet nous considérons que les preuves produites par la recherche devraient trouver leur place aux côtés d'autres types de preuves dans tout projet de transformer les politiques et les pratiques éducatives. II ne s'agit donc pas pour nous, dans ce moment de distanciation critique, de nier l'importance à accorder à la preuve dans le développement de nouvelles politiques éducatives ou de nouveaux modes d'interventions éducatives, mais bien de questionner l'évidence selon laquelle celles-ci doivent être basées sur la preuve : de quel type de preuve s'agit-il ? Quel rôle joue-t-elle dans le jugement politique ou le jugement professionnel de l'enseignant ? Comment est-elle articulée avec d'autres types de preuves dans le travail du politique ou du professionnel de l'enseignement ? Comment son utilisation estelle conçue ? Bref, en affirmant notre foi en une forme de rationalité scientifique, nous tentons d'éviter de tomber dans le piège tendu par la rhétorique de I'EBP affirmant que toute mise en question de la thèse selon laquelle la preuve doit fonder les politiques et pratiques éducatives ne peut être qu'irrationnelle et le fait de personnes dont le jugement est obscurci par l'idéologie ou toute autre forme d'opinions non fondées sur la raison. En fait, dans cette partie, nous adoptons la même position que celle qui est valorisée dans le champ de la médecine basée sur des preuves: les savoirs issus de la recherche doivent trouver leur place aux côtés d'autres formes de savoirs dans le raisonnement professionnel. En ce qui nous concerne, nous formulons l'hypothèse d'analyse que le principal danger que court le mouvement EBP, c'est de verser dans une forme d'orthodoxie.

\section{Maintenir le lien entre logique intégrative et logique agrégative}

II y a plusieurs manières de représenter l'évolution du champ anglo-américain de recherche concernant l'analyse de la politique éducative. Dans ce qui suit, nous allons surtout nous appuyer sur le point de vue de Scribner, Aleman et Maxcy (2003) concernant l'évolution du champ de la politique éducative et de son analyse. En ce qui concerne l'évolution de la politique éducative aux États-Unis, la thèse de Scribner et ses collègues (2003) est la suivante. Tout au long de I'histoire de l'éducation américaine, il y aurait eu à l'œuvre et en tension deux modes de gouvernance des institutions éducatives : un mode intégratif et un mode agrégatif ou pluraliste. Le premier mode est élitiste et centré sur la définition et la poursuite d'un bien commun éducatif par un groupe particulier (familièrement nommé WASP ou White Anglo-Saxon protestant). Le second mode reconnaît la pluralité des intérêts des divers groupes concernés et cherche à leur faire une place légitime et à 
les accommoder dans une sorte de régime de marché concurrentiel. C'est ainsi que le common school movement du XIX siècle (l'éducation publique pour tous faisant la promotion d'une culture nationale - le patriotisme constitutionnel -, d'une éthique du travail protestante et des principes de l'ordre social américain) et le progressive reform ideology de la première moitié $\mathrm{du} \mathrm{xx}^{\mathrm{e}}$ siècle (application du management scientifique à l'éducation, institutionnalisation de la méritocratie, cf. Callahan, 1962) auraient participé d'une logique intégrative, alors qu'après la deuxième guerre mondiale, le mouvement des droits civiques et le militantisme syndical des enseignants auraient poussé la gouvernance vers un mode plus agrégatif et pluraliste. On aura compris que le mode intégratif peut mener à une forme d'hégémonie, d'orthodoxie, de hiérarchisation ou d'exclusion, dérives que le mode agrégatif cherche à combattre. Ce second mode, plus ouvert et diversifié, risque par contre de faire perdre au champ sa cohérence ou sa consistance, en le transformant en une sorte d'auberge espagnole.

Selon Scribner et ses collègues (2003), les modes intégratif et agrégatif caractérisent également l'évolution du champ de l'analyse de la politique en éducation. L'histoire de ce champ serait marquée par des périodes plus intégratives ou plus agrégatives d'unité autour d'un noyau central ou de diversité et de cohabitation plus ou moins conflictuelle entre des courants et des approches : " le développement du champ reflète la lutte entre le désir d'un champ d'étude intégré et discipliné et le désir d'un champ large et inclusif à la recherche de nouveaux problèmes, de nouvelles théories et de nouvelles méthodes [...] Cette nécessaire et productive tension est cruciale pour l'avancement du champ » (Scribner et al., 2003 , p. 15). Divers courants se disputent la place centrale du champ et cherchent à incorporer et à dépasser les autres. Si l'évolution du champ angloaméricain de l'analyse de la politique en éducation est marquée par la tension et le jeu d'équilibre entre une logique de type intégratif et une logique de type agrégatif, la reconnaissance des courants, de leurs forces et de leurs faiblesses, et leur mise en dialogue constituent, selon Scribner et ses collègues (2003), la meilleure stratégie collective de construction d'un noyau conceptuel du champ de l'analyse de la politique en éducation riche et dynamique, c'est-à-dire d'une forme d'intégration qui incorpore la diversité et la complexité au lieu de l'éliminer. Retenons pour notre propos cette tension entre l'intégration et l'agrégation, entre l'unité et la diversité, entre l'hégémonie et la hiérarchie d'une part, et la concurrence et la complémentarité d'autre part. Ce sont des éléments utiles pour construire un cadre d'analyse de la dynamique interne du champ disciplinaire de l'éducation et comprendre l'émergence et l'institutionnalisation de l'EBP.

Sur cette base analytique, nous souhaitons soulever deux questions concernant l'approche de l'EBP. En effet, cette approche semble renouer avec les orientations initiales du champ. Tout d'abord, dans la perspective d'un optimisme raisonné, ne traduit-elle pas un retour à la quête de "scientificité " (ou « méthodologisme ") caractéristique des débuts du champ, d'une stratégie de reconnaissance du champ et du bien-fondé de sa contribution à la décision politique ? Ensuite, l'EBP ne participe-t-elle pas de la logique intégrative en tentant de réduire la complexité du champ par la mise à plat les divers courants, les réduisant à des répertoires de variables pertinentes que doit incorporer l'étude scientifique construite suivant la règle d'or de la recherche ? Grâce à cette méthodologie, le poids relatif des variables d'intérêt personnel et des contextes micropolitiques locaux, de même que celui des variables associées aux réalités institutionnelles et culturelles dominantes, seront connus avec précision, mettant fin à des débats ainsi vidés de leur sens et contribuant par là même à une prise de décision rationnelle et fondée sur une preuve "solide et indiscutable". 
II apparaît que les vertus de la logique intégrative sont de contribuer à maintenir une forme d'unité dans le champ autour des problèmes abordés, de la trame théorique dans laquelle les mailler et des méthodologies utilisées, ce qui assure une forme d'accumulation des résultats au sein de la discipline et de réduction de l'impression d'éclatement et de complexité du champ pour les personnes qui lui sont extérieures. Ce sont de telles vertus qui sont attribuées à l'EBP par Hargreaves (1996) dans sa charge contre la recherche en éducation. En effet cet auteur déplore le manque d'unité du champ de la recherche en éducation : il s'agit d'un champ éclaté où il est difficile pour quelqu'un qui l'intègre de se forger une vue d'ensemble (voir Hammersley, 2002 pour une analyse de l'histoire de cet argument dans la pensée de Hargreaves). II s'agit d'un champ où la recherche évolue davantage en référence à des modes que par rapport au fait que les problèmes ont été résolus. II s'agit d'un champ où l'accumulation du savoir est faible, voire inexistante : les recherches s'appuient peu sur les travaux antérieurs, procèdent rarement à la réplication ou à un travail de réfutation et de raffinement des modèles. II s'agit d'un champ éclaté en une myriade d'îlots intellectuels non reliés. Bref, une sorte d'auberge espagnole selon les détracteurs de la recherche en éducation.

Inversement, les vertus de la logique agrégative sont de contribuer au renouvellement de la manière dont les problèmes sont posés dans le champ et, de la sorte, de favoriser l'émergence de nouveaux schèmes d'intelligibilité et de nouvelles méthodologies, et de contribuer au développement d'une approche multiréférentielle des politiques de l'éducation ainsi qu'à l'établissement d'un climat propice à un débat entre les différentes approches. La logique agrégative participe de la sorte à une meilleure prise en compte de la complexité de la chose éducative et introduit une exigence de modestie dans tout effort de modélisation du réel. C'est le point de vue soutenu par Phillips (2007) et par Scribner et ses collègues (2003). Pour le premier, un philosophe des sciences, il apparaît difficile de n'avoir qu'une et une seule définition opératoire de l'activité scientifique ; il vaut mieux selon lui convenir d'une famille de pratiques diversifiées parfois convergentes, parfois divergentes, et comportant inévitablement un jugement prononcé par le collectif des scientifiques sur la valeur globale de la (ou des) preuve(s). Scribner et ses collègues (2003), adoptant un point de vue plus proche de Khun, sont d'avis que le champ de la politique en éducation " a avancé grâce à l'interaction dynamique des paradigmes ou des traditions de recherche, critiquant et se complétant, chacun étant en concurrence pour définir le centre. Le champ a bénéficié et continuera de bénéficier d'un engagement authentique et critique à l'égard d'un éventail large de problèmes, de théories et de méthodes. Nous soutenons que, quoique désordonnée et litigieuse (contestée), cette interaction est centrale pour le champ " (Scribner et al., 2003, p. 33). Comme nous l'avons souligné précédemment, les rapports entre ces deux logiques jouent un rôle structurant dans le champ, le prémunissant des maux engendrés par une forme extrême d'orthodoxie ou d'éclatement.

Doit-on craindre, avec l'institutionnalisation de I'EBP, le développement d'une forme d'orthodoxie ? Les défenseurs de l'EBP adoptent généralement un discours politiquement correct concernant le pluralisme intellectuel et méthodologique. Ainsi Slavin (2002, p. 18) affirme : “ Je devrais m'empresser de dire que des formes de recherche autres qu'expérimentales, aléatoires ou couplées, peuvent être aussi d'une grande valeur. La recherche corrélationnelle et descriptive est essentielle à l'élaboration théorique et pour suggérer des variables devant être incorporées dans les expérimentations [...] Cependant, l'expérimentation est le modèle de choix pour des études qui ambitionnent de formuler des conclusions causales, et particulièrement pour des évaluations éducatives ». En Angleterre, Hargreaves 
(1999) a introduit l'expression : “pratique informée par la preuve » au lieu de "pratique fondée sur la preuve", afin d'accorder une place au jugement professionnel des enseignants.

Par contre, certains chercheurs de la mouvance critique perçoivent l'EBP comme l'instrument d'une volonté politique d'étouffer les épistémologies alternatives ainsi que de disqualifier des formes d'activité savantes contribuant à développer des lectures alternatives du social et ayant porté des fruits en matière de développement de justice sociale et d'équité (Lincoln \& Cannella, 2004). D'ailleurs, pour Lather (2004), les efforts fédéraux américains pour légiférer en matière de " scientificité » de la recherche en éducation constituent une tentative de lutter contre la prolifération des approches. NCLB prescrit une conception étroite de la " scientificité » (Shaker \& Ruitenberg, 2007) et contribue à définir une forme de savoir officiel en éducation (Bloch, 2004), un régime de vérité (Lincoln \& Canella, 2004).

Les politiques fédérales américaines confèrent de cette façon une forme de souveraineté épistémologique à une conception post-positiviste de la science (Bloch, 2004), reposant sur l'assertion selon laquelle la science est une activité strictement rationnelle dont l'objectivité découle de la qualité de ses méthodes, une activité située en dehors de tout rapport avec des intérêts politiques et personnels (Lincoln, 2004). À la suite de l'adoption du NCLB et de la publication du rapport du National research council ou encore de l'ouvrage de Boruch et Mosteller (2002), Lather (2004), Bloch (2004) et Lincoln (2004) n'hésitent pas à évoquer la fin de la détente et la reprise de la guerre des paradigmes. Dans cette perspective, l'EBP apparaît comme un instrument de réorientation de la recherche en éducation aux États-Unis et ailleurs dans le monde (Lather, 2004). Elle constitue une manière de contrôler la recherche sur les politiques éducatives et les modes d'intervention pédagogique, ainsi que de réserver l'accès aux ressources disponibles pour la recherche à ceux qui croient en la preuve expérimentale et qui maîtrisent cette approche. Elle contribue à la mise en place d'une oligarchie de chercheurs, à marginaliser les discours issus de modèles alternatifs d'exercice de l'activité scientifique, voire même à étouffer la contestation et le débat (Lincoln, 2004). Sans nécessairement partager l'intégralité de cette analyse, différents auteurs plus modérés dans la critique (Hammersley, 2005 ; Hodkingson, 2008 ; Oancea, 2005) utilisent le vocable de nouvelle orthodoxie pour qualifier l'EBP. De cette manière, ces auteurs ne cherchent pas à disqualifier l'EBP, mais à cerner certains des enjeux liés à son institutionnalisation, ainsi que certaines de ses dérives potentielles. Bref, le risque d'une rupture de la tension entre les deux logiques est réel et doit être pris en compte.

\section{Les rapports entre science, politique et pratique}

La critique de l'EBP porte très souvent sur la conception des rapports entre recherche, politique et pratique sous-jacente à cette approche. Différentes métaphores sont utilisées pour cerner ces rapports et certains de leurs enjeux : "savant vs technicien " (scholar vs technician d'après Lynd cité par Hargreaves, 1999), modèle des Lumières vs modèles d'ingénierie (enlightenment vs engineering models, d'après Janowitz cité par Hargreaves, 1999 et Hammersley, 2002), rôle culturel vs rôle technique de la recherche (cultural role vs technician role of research), vision instrumentale vs définition de la situation (instrumental view vs definition of the situation, d'après Kennedy, 1999). Outre le fait que l'origine de ces métaphores indique que la question des rapports entre recherche, politique et pratique est une question récurrente voire même centrale dans le champ des sciences sociales, elles permettent de mettre en lumière ce qui est visé : une 
conception linéaire du changement sous-jacente à l'EBP. Ces différentes catégories se recoupent partiellement autour de deux fonctions distinctes et complémentaires de la recherche. De manière très schématique, les images du savant, des Lumières ou du rôle culturel de la recherche réfèrent à la fonction d'intelligibilité de la recherche. Celle-ci a pour rôle de produire de nouveaux schémas d'intelligibilité. Elle contribue de manière indirecte au développement des politiques et des pratiques. Elle façonne les cadres interprétatifs des personnes et nourrit leur jugement et leur compréhension du monde. Dans cette perspective, ce n'est pas la preuve qui importe, c'est le concept !

Les images du technicien et de l'ingénieur appuient plutôt une fonction instrumentale de la recherche. Celle-ci a pour fonction de développer des solutions techniques applicables par les politiciens et les praticiens. Elle contribue donc directement au développement des politiques et des pratiques en produisant des réponses claires à des questions posées en termes de rapports moyens-fins : quel est le procédé le plus efficace pour produire un effet ? Le débat concernant l'EBP manifeste de la sorte une forme de proximité avec la manière dont certains (Langemann, 2000) ont tenté de poser l'opposition entre Dewey et Judd au début du $\mathrm{xx}^{\mathrm{e}}$ siècle, lors de la constitution du champ de l'éducation aux États-Unis. De manière très schématique et caricaturale, on peut résumer ce débat à deux conceptions opposées de la manière dont la science est en mesure de façonner les modes d'intervention éducatives : directe et indirecte. En effet, toujours de manière très schématique, Judd concevait que la science pouvait contribuer directement à l'intervention éducative selon le modèle de la science appliquée. Dewey quant à lui concevait que la contribution des sciences à l'éducation ne pouvait opérer que de manière indirecte, médiatisée en quelque sorte par l'activité d'investigation, cette forme de délibération dans laquelle s'engage la personne lorsqu'elle est confrontée à une situation douteuse.

De manière plus concrète, cela revient à se placer à l'un ou l'autre des deux pôles suivants, ici formulés de manière interrogative : les problèmes éducatifs sontils « techniques ", soumis à la rationalité instrumentale et à une ingénierie politique ou comportent-ils des dimensions normatives, inévitablement conflictuelles dans une société moderne avancée, à propos des moyens et des fins ? Dans le premier cas, le savoir scientifique qui dégage des relations de causalité est le seul à même de fonder la décision, et la valeur de référence y est l'efficience. Dans le second cas, la rationalité est " pratique ", c'est-à-dire qu'elle incorpore des considérations de valeurs, de finalités et de choix éthiques et moraux. Outre le savoir scientifique, le savoir tacite et expérientiel des praticiens doit être pris en considération et la valeur de référence y est le caractère approprié et raisonnable de l'action. De nombreux travaux concernant la nature du travail enseignant ont mis en lumière sa nature pratique plutôt que technique (Hammersley, 2002). Ce travail est fondé pour une part sur une forme de jugement qui a quelque chose à voir avec la phronésis d'Aristote (Biesta, 2007 ; Sanderson, 2006), une forme de sagesse (Shulman, 1987). Aussi, le jugement professionnel des enseignants est davantage orienté par des questions de valeurs que par des questions techniques. D'ailleurs, au quotidien, l'enseignant en salle de classe est moins confronté à des problèmes à résoudre qu'à des dilemmes à dépasser (Tardif \& Lessard, 1999). La thèse développée par différents auteurs (Hammersley, 2002 ; Biesta, 2007 ; Sanderson, 2006) est que l'EBP néglige cette caractéristique du jugement professionnel des enseignants. Elle est plutôt basée sur une vision de l'enseignant comme un technicien dont le travail reposerait sur le suivi de règles d'action déduites de la recherche. En langage ergonomique, l'EBP est du côté de la conception de la tâche, mais néglige l'activité. Si l'on revient à la métaphore des Lumières, la fonction de la recherche est 
de créer les conditions intellectuelles en mesure de changer la compréhension que les praticiens (ou les politiques) ont du problème, de produire des connaissances pour nourrir les délibérations pratiques plutôt que de développer des solutions techniques directement applicables. Ce point de vue se veut aussi en lien avec un modèle de professionnalisation du métier, celui du praticien réflexif. Bref, le débat entre une contribution directe ou indirecte de la recherche à la pratique est relancé. Nous défendons plutôt l'idée d'une contribution indirecte.

\section{Le passage de la conception d'une réforme à sa mise en œuvre}

Les positions adoptées par les uns et les autres dans ce débat sur le type de recherche valable en éducation influent aussi sur la manière de concevoir la politique éducative et sa mise en œuvre. En effet, dans une vision instrumentale des rapports entre recherche et politique, cette dernière apparaîtra surtout comme un input, exogène au système qu'elle est censée modifier. Elle ne sera pas saisie comme nécessairement enchâssée (ou embedded) dans les différents niveaux du système touché. Dans le premier cas de figure, la mise en œuvre est en général et doit être un processus linéaire, du haut vers le bas, de formation des acteurs subalternes à la bonne pratique et de fidélité des différents niveaux du système au modèle extérieur de départ. Dans le second cas de figure, l'implantation des politiques apparaît comme une réalité multidimensionnelle et multiniveau. À chacun des niveaux organisationnels, la politique est connectée à divers systèmes d'action verticaux et horizontaux qui l'incorporent et influent sur elle (ses intentions, ses ressources attachées, ses cadres régulateurs, ses échéanciers, voir Spillane, 2004). Aussi, deux visions des acteurs s'affrontent : sont-ils des acteurs aux connaissances limitées et incompétents, à qui il faut fournir les connaissances et dont il faut développer les compétences nécessaires à l'implantation fidèle de la politique ? Ou au contraire possèdent-ils l'expérience, le savoir plus ou moins explicite et les outils sociocognitifs nécessaires pour participer à des processus de construction de sens (ou sensemaking) dans lesquels leurs valeurs, leurs croyances, leurs compréhension et savoirs (des éléments institutionnels) jouent un rôle déterminant ?

Tyack et Cuban (1995) ont proposé que soit reconnue comme légitime et nécessaire ce qu'ils ont appelé l'hybridation des politiques et des réformes, ce qui suppose un travail de «traduction ", d'« interprétation » et d'opérationnalisation, voire de révision et de transformation des politiques et des réformes au fur et à mesure qu'elles s'éloignent de la sphère politique pour se rapprocher de la sphère technique, c'est-à-dire du travail réel effectué par des enseignants dotés d'une " agentivité ", c'est-à-dire de connaissances et de compétences. En effet, ces auteurs estiment que trop souvent, tout se passe comme si les chercheurs pensaient que les réformes sont bien construites et rationnelles, comme si la conception précédait et déterminait l'implantation, qui ne serait que la mise en œuvre relativement technique par des exécutants d'“ en bas " de ce qui a été préalablement décidé « en haut " par les élites pensantes (la "noosphère "). Dans cette vision, le sens d'une réforme ou d'une politique est prédéterminé et univoque et tous les acteurs doivent y adhérer sans discussion, sans appropriation, sans médiation, sans transformation ou évolution.

Toujours selon Tyack et Cuban (1995), il serait plus juste d'envisager une réforme comme un projet de changement qui doit nécessairement être transformé au fur et à mesure de sa réalisation, surtout lorsque celle-ci comporte une modification de représentation et de pratique des acteurs. Non seulement ce processus d'hybridation est-il inévitable, mais il est nécessaire dans un secteur institutionnel où la « technologie » de base est peu développée et repose essentiellement 
sur des rapports sociaux et sur leur signification négociée et partagée (Tardif \& Lessard, 1999). L'hybridation est fort probablement une bonne chose, surtout si elle est soutenue et accompagnée, rendant légitime une appropriation par les acteurs qui repose sur un processus de construction du sens à propos du changement par ceux-là même qui doivent le mettre en œuvre, construction qui, Iorsqu'elle est réussie, renouvelle (au sens de redonner de la vigueur et aussi d'y introduire du nouveau) leur pratique. Cette hybridation implique des allers-retours entre l'élaboration et la mise en œuvre de la politique, entre la politique et la pratique, et des boucles de rétroaction efficaces. Elle est facilitée par un pilotage négocié du changement (Perrenoud, 1999). Certes, ces oppositions sont relatives mais elles n'en sont pas moins utiles pour comprendre les difficultés de nombreuses mises en œuvre de politiques en éducation. Elles aident à comprendre aussi la valeur heuristique de la notion d'hybridation des politiques proposées par Tyack et Cuban (1995) et à concevoir la réussite en matière de politique éducative à l'intérieur du paradigme de la complexité et de l'apprentissage. Est-ce uniquement la fidélité au modèle de départ ? Est-elle fonction de la durabilité des changements souhaités et des effets désirés ? Est-ce la capacité du système et de ses acteurs de maintenir, d'étendre et d'approfondir une action valorisé ? Est-ce l'apprentissage systémique?

Selon Ogawa (2004), les politiques éducatives américaines actuelles et le courant de recherche qui les soutient considèrent l'incertitude comme problématique et comme devant être réduite, voire éliminée complètement. Ces recherches essaient donc d'identifier, de nommer et de modéliser des processus et des qualités qui sous-tendent l'efficience de l'enseignement indépendamment des "settings » et des enseignants singuliers. Pourtant, selon Clark (1998, cité par Ogawa, 2004, p. 34), la recherche sur le savoir et la pensée des enseignants «ne promet pas de découvrir une méthode générique efficace ou un ensemble de techniques pour gérer l'incertitude, la complexité ou des dilemmes. De par leur nature même, ces qualités défient la quête d'une solution technique ". Ogawa en conclut que si l'incertitude ne peut être totalement évacuée de la technologie de base de l'enseignement, alors il faut penser l'organisation scolaire de telle sorte qu'elle puisse contribuer à l'accroissement des connaissances et des compétences des enseignants, ce qui est une manière de dire qu'il faut transformer les établissements en organisation apprenante ou faciliter l'insertion des enseignants dans des réseaux et des communautés professionnelles d'apprentissage. Cela signifie que la « culture » est plus importante que la "structure " organisationnelle, qu'il est important de faciliter pour les acteurs de la base (les enseignants et les directeurs d'école) l'accès et la capacité d'influencer les autres sous-systèmes de l'organisation et que l'ordre et le désordre, la prescription précise et l'incertitude doivent cohabiter. Cela est en définitive une condition essentielle de l'apprentissage, selon Weick et Westley (1996, p. 441) qui insistent sur la nécessaire juxtaposition de l'ordre et du désordre afin qu'un espace d'apprentissage soit possible. On le constate, le néo-institutionnalisme d'Ogawa est proche des approches centrées sur les acteurs et le « sensemaking " des communautés professionnelles d'apprentissage (Weick, 1995).

II nous semble que la piste de l'apprentissage organisationnel est fort intéressante, car elle permet de prendre en compte toute la complexité de la rationalité pratique à l'œuvre dans l'enseignement. Elle soulève par ailleurs des questions qui méritent d'être étudiées par les chercheurs du champ de la politique en éducation. Si la réussite d'une politique éducative est liée à l'apprentissage organisationnel, comment apprend-on de son expérience et de celles de ses collègues ? Comment l'élaboration des politiques peut-elle générer et utiliser le savoir profes- 
sionnel de manière congruente avec les finalités des réformes ? Comment relier l'apprentissage individuel et l'apprentissage organisationnel ? Comment intégrer ce type d'apprentissage dans les processus d'élaboration et d'implantation des politiques et des réformes ? Comment l'administration de l'éducation peut-elle intégrer l'apprentissage dans le fonctionnement des établissements ?

\section{CONCLUSION}

Le mouvement de l'EBP, tel qu'il se développe en Angleterre et aux ÉtatsUnis, a un effet structurant sur la recherche en éducation. En effet, il propose pour celle-ci des orientations pragmatiques claires : la recherche doit contribuer à l'amélioration de la qualité de l'éducation, c'est-à-dire de son efficacité et de son efficience. Elle doit répondre aux questions et aux enjeux politiques et professionnels ; ces questions sont formulées par l'instance politico-administrative et par les professionnels en exercice. Les réponses doivent être formulées en termes de pratiques exemplaires, de règles pour l'organisation scolaire et de politiques institutionnelles. Les chercheurs, qui doivent partager la définition des questions et des enjeux avec les acteurs ci-dessus mentionnés, doivent répondre à ces questions en utilisant deux méthodologies de recherche : les essais contrôlés randomisés et la revue systématique des écrits et des données quantitatives (métaanalyse). Ainsi conçue, la recherche en éducation prend un virage résolument " partenarial » et " pratique ». Partenarial en ce sens que les chercheurs ne sont pas les seuls à orienter et réguler l'effort de recherche. L'autonomie du champ universitaire de la recherche en éducation s'en trouve considérablement réduite, au profit des instances " extérieures » au champ qui en assurent le financement et une part non négligeable de la légitimité. Virage "pratique ", dans la mesure où cette recherche trouve sa raison d'être dans la délimitation du noyau technique capable d'assurer l'efficacité de l'intervention éducative. Ce virage partenarial et pratique est censé être l'occasion d'une plus grande cohérence et cohésion de l'effort de recherche, contribuant ainsi à en limiter la dispersion et l'éclatement. Pour nous, dans un champ professionnel comme l'éducation, ces orientations ne sont pas illégitimes. Ce qui pose problème ce sont une vision réductrice des métiers de l'éducation, techniciste et vidée de ses aspects «pratiques » au sens où nous l'entendons dans ce texte, ainsi que la volonté d'imposer une et une seule méthodologie (essais contrôlés randomisés). Aussi, l'EBP ne risque pas de contribuer à une théorisation plus forte du champ et de ses sous-champs constitutifs. Car à se soucier de ce qui fonctionne et produit les effets souhaités, on s'attarde peu à comprendre pourquoi les effets se produisent et sont mesurés. L'EBP valorise la recherche évaluative : celle-ci s'accommode fort bien d'une sous-théorisation de ses objets, pourvu que les «faits " (les «big facts » dégagés des revues systématiques des recherches empiriques construites selon la règle d'or des essais randomisés contrôlés) parlent haut et fort. Dans un domaine qui demeure fortement concurrencé par le sens commun, la sous-théorisation des objets de recherche en éducation nous apparaît problématique en tant que stratégie de développement et de reconnaissance.

Frédéric Saussez Frederic.Saussez@Sherbrooke.ca CRIFPE, université de Sherbrooke

Claude Lessard

CRIFPE, université de Montréal 
(1) Pour plus de renseignements, vous pouvez consulter le site Internet disponible à l'adresse : <http://www cemcentre.org/RenderPage.asp?LinkID=30324000> (consulté le 8 décembre 2009).
(2) Pour plus de renseignements, vous pouvez consulter le site Internet disponible à l'adresse : <http://www.cemcentre.org/renderpage.asp?linkID=30317000> (consulté le 8 décembre 2009).

\section{BIBLIOGRAPHIE}

ANDERSON S. (2006). "The school district role in educational change: taking stock of what we know ». International journal of educational reform, vol. $15, \mathrm{n}^{\circ} 1$.

ATKINSON E. (2000). "In defense of ideas, or why "what works" is not enough ". British journal of sociology of education, vol. $21, \mathrm{n}^{\circ} 3$, p. 317-330.

BECK U. (2001). La société du risque. Sur la voie d'une autre modernité. Paris : Flammarion.

BENNETT W. (1986). What works: research about teaching and learning. Washington: Department of education.

BIESTA G. (2007). «Why what works won't work: evidencebased practice and the democratic deficit in educational research ». Educational theory, vol. 27, n 1, p. 1-22.

BLOCH M. (2004). «A discourse that disciplines, governs, and regulates: the national research council's report on scientific research in education ". Qualitative inquiry, vol. $10, n^{\circ} 1$, p. $96-110$.

BORUCH R. \& MOSTELLER F. (2002). « Overview and new directions ". In F. Mosteller \& R. Boruch (dir.), Evidence matters. Randomized trials in education research. Washington : The Brookings institution.

BORUCH R., MOYA D. de \& SNYDER B. (2002). « The importance of randomized fields trials in education and related areas ". In F. Mosteller \& R. Boruch (dir.), Evidence matters. Randomized trials in education research. Washington : The Brookings institution.

BOURDONCLE R. (1993). "L'évolution des sciences de l'éducation dans la formation des enseignants en Angleterre ». Revue des sciences de l'éducation, vol. 19, $\mathrm{n}^{\circ} 1$, p. 133-151.

CALLAHAN R. (1962). Education and the cult of efficiency. Chicago : university of Chicago Press.

CAMPBELL D. (1969). «Reforms as experiment ». American psychologist, vol. 24, p. 409-429.

CAMPBELL D. \& STANLEY J. (1963). « Experimental and quasi experimental designs for research ». In N. Gage (dir.), Handbook of research on teaching. Chicago : Rand Mc Nally.

DAVIES P. (1999). "What is evidence-based education? » British journal of educational studies, vol. $47, \mathrm{n}^{\circ} 2$, p. 108-121.

DAVIES P. (2004). "Systematic reviews and the Campbell collaboration ». In G. Thomas \& R. Pring (dir.), Evidencebased practice in education. Corwall : Open university Press.

DAVIES H., NUTLEY S. \& SMITH P. (2000). What works: evidence-based policy and practice in the social services. Bristol : Policy Press.

DEHUE T. (2001). «Establishing the experimenting society: the historical origin of social experientation according to the randomized controlled design ". American journal of psychology, vol. 114, $\mathrm{n}^{\circ} 2$, p. 283-302.

DUC G. (2001). " Les méfiances du clinicien à l'égard de l' "evidence-based medecine" ". Forum médical suisse, vol. $26, n^{\circ} 6$, p. 692-693.

EISENHART M. \& TOWNE L. (2003). « Contestation and change in national policy on "scientifically-based" education research ». Educational researcher, vol. $32, \mathrm{n}^{\circ} 7$, p. 31-38.

ERAUT M. (2004). «Practice-based evidence ». In G. Thomas \& R. Pring (dir.), Evidence-based practice in education. Corwall : Open university Press.

ÉTATS-UNIS : NATIONAL COMMITTEE ON EXCELLENCE IN EDUCATION (1983). A nation at risk. Washington : Department of education.

ÉTATS-UNIS : DEPARTMENT OF EDUCATION (2001). No child left behind act. Washington : Department of education. Disponible sur Internet à l'adresse suivante : <http://www.ed.gov/policy/elsec/leg/esea02/index. html> (consulté le 8 décembre 2009).

ÉTATS-UNIS : DEPARTMENT OF EDUCATION (2002a). The secretary's first annual report on teacher quality. Washington: Department of education.

ÉTATS-UNIS : DEPARTMENT OF EDUCATION (2002b). Strategic plan 2002-2007. Washington : Department of education. Disponible sur Internet à l'adresse : <http:// www.ed.gov/about/reports/strat/plan2002-07/plan. pdf $>$ (consulté le 8 décembre 2009).

EVANS J. \& BENEFIELD P. (2001). "Systematic reviews of educational research: does the medical model fit? " British educational research journal, vol. $27, \mathrm{n}^{\circ} 5$, p. 527-541.

FEUER M., TOWME L. \& SHAVELSON R. (2002). «Scientific culture and educational research ». Educational researcher, vol. 31, n 7 , p. 4-14.

FITZ-GIBBON C. (2003). «Milestones en route to evidencebased policies ». Research papers in education, vol. 18, $\mathrm{n}^{\circ} 4$, p. 313-329.

GIBBONS M., LIMOGES C., NOWOTNY H. et al. (1994). The new production of knowledge. The dynamics of science and research in contemporary societies. Londres : Sage.

GIDDENS A. (1994). Les conséquences de la modernité. Paris : L'Harmattan.

GOUGH D. (2004). «Systematic research synthesis ». In G. Thomas \& R. Pring (dir.), Evidence-based practice in education. Corwall : Open university Press.

GOUGH D. (2007). «Weight of evidence: a framework for the appraisal of the quality and relevance of evidence ". Research papers in education, vol. 22, $\mathrm{n}^{\circ} 2$, p. 213228. 
HAMMERSLEY M. (2001a). «On sytematic reviews of literatures: a narrative response to Evans and Benefield ». British educational research journal, vol. 27, $\mathrm{n}^{\circ} 5$, p. 543-554.

HAMMERSLEY M. (2001b). Some questions about evidencebased practice in education. Symposium on evidencebased practice in education, annual conference of the British educational research association, Leeds, Angleterre. Disponible sur Internet à l'adresse suivante : $<$ http://www.leeds.ac.uk/educol/documents/00001819. htm> (consulté le 8 décembre 2009).

HAMMERSLEY M. (2002). Educational research. Policymaking and practice. Londres : Sage.

HAMMERSLEY M. (2005). «Is the evidence-based practice movement doing more good than harm? Reflections on lain Chalmers' case for research-based policy making and practice». Evidence \& policy, vol. 1, $\mathrm{n}^{\circ} 1$, p. 85-100.

HARGREAVES D. (1996). Teaching as a reserach-based profession: possibilities and prospects. Londres : TTA.

HARGREAVES D. (1997). «In defence of research for evidence-based teaching: a rejoinder to Martyn Hammersley ». British educational research journal, vol. 23 , $\mathrm{n}^{\circ} 4$, p. 405-419.

HARGREAVES D. (1999). « Revitalising educational research: lessons from the past and proposals for the future ". Cambridge journal of education, vol. 29, $\mathrm{n}^{\circ} 2$, p. $405-$ 419.

HILLAGE J., PEARSON R., ANDERSON A. \& TAMKIN P. (1998). Excellence in research on school. Londres : DFEE.

HODKINGSON P. (2008). « Scientific research, educational policy and educational practice in the United Kingdom: the impact of the audit culture on further education " . Cultural studies-critical methodologies, vol. $8, \mathrm{n}^{\circ} 3$, p. 303-324.

HURSCH D. (2007). "Assessing "No child left behind" and the rise of neoliberal education policies ". American educational research journal, vol. 44, $\mathrm{n}^{\circ} 3$, p. 493-518.

JAMESON F. (1991). Postmodernism or the cultural logic of late capitalism. Londres : Verso.

KENNEDY M. (1999). «A test of somme common contentions about educational research ». American educational research journal, vol. 36, $n^{\circ} 3$, p. 511-541.

LANGEMANN E. (2000). An elusive science. The troubling history of education research. Chicago : Chicago university Press.

LATHER P. (2004). " This is your father's paradigm: government intrusion and the case of qualitative research in education ». Qualitative inquiry, vol. 10, n 1, p. 15-34.

LESSARD C. (2006). « Le débat américain sur la certification des enseignants et le piège d'une politique éducative evidence-based ". Revue française de pédagogie, $\mathrm{n}^{\circ} 154$, p. 19-31 et Revue des sciences de l'éducation, vol. 32, $\mathrm{n}^{\circ} 1$, p. 31-52 [numéro conjoint].

LESSARD C. (2008). «Recherche et politiques éducatives ». In A. van Zanten (dir.), Dictionnaire de l'éducation. Paris : PUF, p. 580-584.

LESSARD C. \& BRASSARD A. (2006). « La "gouvernance" de l'éducation au Canada : tendances et significations ». Éducation et sociétés, $n^{\circ} 18$.
LESSARD C. \& MEIRIEU P. (2004). L'obligation de résultats en éducation. Canada : Presses de l'université Laval et Paris : De Boeck.

LINCOLN Y. (2004). « Dual review: scientific research in education \& evidence matters: randomized trials in education research ". Academe, vol. 90, $\mathrm{n}^{\circ} 6$. Disponible sur Internet à l'adresse: <http://www.aaup.org/AAUP/ pubsres/academe/2004/ND/BR/ndlinc.htm> (consulté le 8 décembre 2009).

LINCOLN Y \& CANNELLA G. (2004). «Dangerous discourse: methodological conservatism and governement regimes of truth ». Qualitative inquiry, vol. 10, $\mathrm{n}^{\circ} 1, \mathrm{p}$. 5-14.

LIPMAN T. (2000). "Evidence-based practice in general practice and primary care ". In L. Trinder \& S. Reynolds (dir.), Evidence-based practice. A critical appraisal. Londres : Blackwell.

NORMAND R. (2006). "Les qualités de la recherche ou les enjeux du travail de la preuve en éducation ». Éducation et sociétés, vol. 18, $\mathrm{n}^{\circ} 2$, p. 73-91.

NATIONAL RESEARCH COUNCIL (2002). Scientific research in education. Washington: National academies Press.

NATIONAL RESEARCH COUNCIL (2005). Advancing scientific reserach in education. Washington : National academies Press.

OAKLEY A. (2002). "Social science and evidence-based everything: the case of education ". Educational review, vol. 54, n³, p. 277-286.

OAKLEY A. (2004). «Who's afraid of the randomized controlled trial? » In C. Seale (dir.), Social research methods: a reader. Londres : Routledge, p. 261-268.

OANCEA A. (2005). "Criticism of educational research: key topics and levels of analysis ". British educational research journal, vol. 31, $\mathrm{n}^{\circ} 2$, p. 157-183.

OGAWA R. (2004). « Embracing uncertainty: organizing and leading to enhance the knowledgeability and capability of teachers ». In N. Bennett \& L. Anderson (dir.), Rethinking educational leadership, challenging the conventions. Londres : Sage, p. 27-42.

PEILE E. (2000). "Reflection from medical practice ". In L. Trinder \& S. Reynolds (dir.), Evidence-based practice. A critical appraisal. Londres : Blackwell.

PERRENOUD P. (1999). « Le pilotage négocié du changement dans les systèmes éducatifs. " In J. Lurin \& C. Nidegger (dir.), Expertise et décisions dans les politiques de l'enseignement. Genève : Service de la recherche en éducation, cahier $\mathrm{n}^{\circ} 3$, p. 88-103.

PHILLIPS D. (2007). Is this research rigorous and scientific? Is the wrong question - even if the research is rigourous and scientific! Communication présenté au congrès national des sciences de l'éducation du Mexique, Mérida, Yucatan.

PIRRIE A. (2001). " Evidence-based practice in education: the best medecine? » British journal of educational studies, vol. 49, $\mathrm{n}^{\circ} 2$, p. 124-136.

POPKEWITZ T. (1997). «A changing terrain of knowledge and power: a social epistemology of educational research ». Educational research, vol. 26, n 9, p. 18-29.

POWER M. (1997). The audit society. Rituals of verification. Oxford : Oxford university Press.

REYNOLDS S. (2000). "The anatomy of evidence-based practice: principles and methods ». In L. Trinder 
\& S. Reynolds (dir.), Evidence-based practice. A critical appraisal. Londres: Blackwell.

SANDERSON I. (2003). « Is it what works that matters? Evaluation and evidence-based policy making ». Research papers in education, vol. $18, n^{\circ} 4$, p. 331-347.

SANDERSON I. (2006). "Complexity, "practical rationality" and evidence-based policy making " Policy \& politics, vol. 34, $\mathrm{n}^{\circ} 1$, p. 115-132.

SCRIBNER J., ALEMAN E. \& MAXCY B. (2003). « Emergence of the politics of education field: making sense of the messy center ". Educational administration quarteley, vol. $39, n^{\circ} 1$, p. $10-40$.

SHAKER P. \& RUITENBERG. (2007). « Scientifically-based research: the art of politics and the distorsion of science ». International journal of research \& method in education, vol. 30, $\mathrm{n}^{\circ} 2$, p. 207-219.

SHULMAN L. (1987). «Knowledge and teaching: foundations of the new reforms ". Harvard educational review, p. 1-23.

SIMONS H. (2003). «Evidence-based practice: panacea or over promise? ". Research papers in education, vol. 18, $n^{\circ} 4$, p. 303-311.

SLAVIN R. (1995). « Best evidence synthesis: an intelligent alternative to meta-analysis ". Journal of clinical epidemiology, vol. 48, $\mathrm{n}^{\circ} 1$, p. 9-18.

SLAVIN R. (2002). "Evidence-based educational policies: transforming educational practice and research ". Educational researcher, vol. $31, \mathrm{n}^{\circ} 7$, p. 15-21.

SLAVIN R. (2004). « Education research can and must adress what works questions ». Educational researcher, vol. 33, $\mathrm{n}^{\circ} 1$, p. 27-28.

SLAVIN R. (2008a). "What works? Issues in synthesizing educational programs evaluations ». Educational researcher, vol. 37, $\mathrm{n}^{\circ} 1$, p. 5-15.

SLAVIN R. (2008b). " Evidence-based reform in education: which evidence counts? ". Educational researcher, vol. 37, $\mathrm{n}^{\circ} 1$, p. 47-50.

SPILLANE J. (2004). Standards deviation. How schools misunderstand education policy. Cambridge : Harvard university Press.
TARDIF M. \& LESSARD C. (1999). Le travail enseignant au quotidien. Laval : Presses de l'université Laval.

THOMAS G. (2004). «Introduction: evidence and practice ». In G. Thomas \& R. Pring (dir.), Evidence-based practice in education. Corwall : Open university Press.

TOOLEY J. \& DARBY D. (1998). Educational research: a critique. Londres : OFSTED.

TRINDER L. (2000). " Introduction: the context of evidencebased practice ». In L. Trinder \& S. Reynolds (dir.), Evidence-based practice. A critical appraisal. Londres : Blackwell.

TRÖHLER D. (2006). «The new languages and old institutions: problems of implementig new school governance ». In P. Smeyers \& M. Depaepe (dir.), Educational research: why " what works » doesn't work. Dordrecht : Springer.

TROTTIER C. (1987). « La nouvelle sociologie de l'éducation en Grande-Bretagne : un mouvement de pensée en voie de dissolution ? " Revue française de pédagogie, $\mathrm{n}^{\circ} 78$, p. 5-20.

TYACK D. \& CUBAN L. (1995). Tinkering toward utopia, a century of public school reform. Cambridge [Ma.] : Harvard university Press.

WEICK K. (1976). "Educational organizations as loosely coupled systems ". Administrative science quaterly, vol. $21, \mathrm{n}^{\circ} 1, \mathrm{p} .1-19$.

WEICK K. (1995). Sensemaking in organizations. Thousand Oaks [Cal.] : Sage.

WEICK K. \& WESTLEY F. (1996). « Organizational learning: affirming an oxymoron ". In S. Clegg, C. Hardy \& W. Nord (dir.), Handbook of organization studies. Thousand Oaks [Cal.] : Sage, p. 440-458.

WEICK K., SUTCLIFFE K. \& OBSTFELD D. (2005). «Organizing and the process of sensemaking ». Organization science, vol. $16, \mathrm{n}^{\circ} 4$, p. 409-421.

WHITTY G. (2006). «Education(al) research and education policy making: is conflict inevitable? » British educational research journal, vol. $32, n^{\circ} 2$, p. 159-176. 